lescence (K. Lam, Royal Postgraduate Medical School, London). This finding is consistent with the notion that EBV infection, together with malaria-associated T-cell immunosuppression, provides an exceptional reservoir of infected B cells in which a c-myc translocation could occur. The $T$-cell response affected by malaria is apparently directed at several of the viral genes expressed in cells immortalized in vitro. Cytotoxic T-cell clones that recognize B cells immortalized only by strain A of EBV, but not by strain $B$, have been shown to recognize two viral proteins (EBNA-2 and EBNA-3A) found in the nucleus. These findings come from experiments performed by introducing expressed viral genes from strain $\mathrm{A}$ that encode these nuclear proteins into B cells immortalized by strain B that are not recognized by the T-cell clones and finding that the recipient $B$ cells subsequently are targets for killing (D. Moss, Queensland Institute of Medical Research). As long as these gene-transfer experiments do not affect the expression of relevant surface molecules of the major histocompatibility complex, they indicate that T-cell recognition of EBV-infected cells could be similar to the recognition of influenzainfected cells ${ }^{4}$. Biopsies of Burkitt's lymphomas often do not express EBNA-2 or EBNA $-3 A^{3}$ and thus could escape the $T$-cell recognition described by Moss.

The expression of EBNA-2 in cells has been found to increase the expression of another viral protein, latent membrane protein, which itself can induce the expression of several activation antigens (S.D. Abbot, University of Birmingham; E. Kieff, Harvard Medical School). This protein is often not found in biopsies of Burkitt's lymphomas ${ }^{3}$, in agreement with the failure of these lymphomas to express B-cell activation antigens.

A candidate for a cell in which c-myc translocation to an immunoglobulin locus can occur has been identified as an EBVimmortalized pro-B cell derived initially by infection of cells from fetal liver ${ }^{5}$. These cells yield different translocations involving the immunoglobulin $\mathrm{H}$ locus on propagation in culture (E. Altiok, Karolinska Institute). They also express B-cell activation antigens and at least seven EBV proteins. If they do represent the virusinfected precursor of a Burkitt's lymphoma, then development of this tumour will probably involve the loss of expression of activation antigens and some viral proteins during differentiation.

This proposed complex role for EBV in carcinogenesis will require further diverse tests before it serves as a thesis to be reconciled with new and differing views. Evidence for the association of the virus with B-cell lymphomas in transplant recipients and in people with AIDS has accumulated and indicates its direct role in carcinogenesis in severely immuno- compromised hosts (D.H. Crawford, Imperial Cancer Research Fund, London; G. Lenoir, IARC, Lyon). By contrast with Burkitt's lymphoma cells, these tumour cells express both activation markers and at least eight viral proteins. This direct role of EBV lends support to the notion that it contributes causally to Burkitt's lymphoma. Its role in Burkitt's lymphoma may eventually be substantiated by testing in people a vaccine that has been found to protect New World monkeys from developing lymphomas following intravenous administration of EBV (M.A. Epstein, University of Oxford).

Bill Sugden is in the McArdle Laboratory for Cancer Research, University of Wisconsin, Madison, Wisconsin 53706, USA.

1. Galloway, J. Nature 338, 463-464 (1989).

2. Knutson, J.C. \& Sugden, B. Adv. Viral Oncol. 8, 151 (1989)

3. Rowe, M. et al. EMBO J. 6, 2743-2751 (1987)

4. Townsend, A.R.M et al Cell 44,959-968 (1986)

5. Otsu, M. et al. Molec. cell. Biol. 7, 708-717 (1987).

\title{
No new fusion under the Sun
}

ELECTROCHEMICALLY induced fusion ${ }^{1,2}$, now a subject of popular debate, is not a new notion. In the 27 April issue of Nature, extracts were printed in the News and Views section ${ }^{3}$ from the debate surrounding the claim during the 1920s of Peters and Paneth $^{4,5}$ that ordinary hydrogen absorbed in palladium fuses to form helium. In his news story in the same issue ${ }^{6}$, Steven Dickman mentioned contemporary work of Tandberg at the Electrolux laboratories in Stockholm, which resembles closely the recent work, involving electro-chemical cells and the release of heat. My father, Torsten Wilner, worked with Tandberg in this and other nuclear research. I still have their notes which are a source of fascinating insights.

Tandberg's introduction to this work was indirect: he started in 1925 by trying to find metals suitable for sealing hydrogen in refrigerators. He became interested in palladium, but for its contrary properties - its permeability and large absorption capacity for hydrogen. He was struck by the work of Paneth and Peters ${ }^{4,5}$, who were attempting to produce helium - for use in airships - by the "spontaneous nuclear catalysis" of hydrogen atoms in palladium metal, and who expected heat to be produced, although they did not pursue this aspect. As noted in Dickman's report ${ }^{6}$, the helium that they identified spectroscopically was in fact atmospheric, not the product of any nuclear reaction.

Tandberg followed up this work, using electrolysis to concentrate hydrogen at the palladium surface - effectively generating a high hydrogen pressure, he argued. The main benefit was to be the generation of energy: the original title of Tandberg's 1927 patent application for the process was " $\mathrm{A}$ method for the release of atomic energy", although this was later altered to "A method for producing helium", possibly as a concession to the patent office.

The patent was not granted, the main reason given being Tandberg's failure to answer the office's objections: what is "hydrogen of highest concentration"?; the mechanisms for the extraction of energy needed better explanation; and the description was not sufficiently complete for a competent person to use the invention.
After the discovery of deuterium in 1932, however, Tandberg and Wilner repeated the experiment, now using heavy water, probably supplied by Niels Bohr.

Their enthusiasm for fusion fired, the two continued with experiments using exploding wires, an approach now used in some laboratories to accelerate ions to sufficiently high energies to overcome Coulomb repulsion. High-voltage capacitors were discharged through thin palladium rods saturated electrolytically with deuterium. On the night of the first discharge, Tandberg asked my father to leave so that he would be able to report what had happened in the event of a nuclear explosion. Nothing spectacular did happen, although the noise of the discharges became a feature of the laboratory routine. No clear-cut results were obtained, possibly because good diagnostic techniques were not then available. I still have the notes and letters referring to the experiments, and some specimens of palladium rods and heavy water. All of these should be studied carefully.

Tandberg and Wilner continued their nuclear experiments in the basement of my father's home. They reproduced the famous experiments of Cockroft and Walton, artificially transforming ${ }^{7} \mathrm{Li}$ into two $\alpha$-particles by acclerated protons. In a subsequent experiment, bombarding a deuteriumsaturated palladium sheet with accelerated deuterons ${ }^{7}$, they observed the fusion reaction of such interest now: $d+d \rightarrow{ }^{3} \mathrm{He}+\mathbf{n}$. The product neutrons were slowed by paraffin wax and detected by the activation of silver. (Much of this work is discussed in a book written to celebrate Tandberg's versatility and achievements ${ }^{8}$.) Bertil Wilner

Bertil Wilner is in the Department of Plasma Physics and Fusion Research, Royal Institute of Technology, S-10044 Stockholm, Sweden.

\footnotetext{
1. Fleischmann, M. \& Pons, S. J. electroanalyt. Chem. 261 301-308 (1989)

. Jones, S.E. et al. Nature 338, 737-740 (1989).

3. Nature 338, 706 (1989)

4. Peters, K. Naturewissenschaften 35, 746-747 (1925)

5. Paneth, F. \& Peters, K. Naturewissenschaften 43, 956$962(1926)$

6. Dickman, S. Nature 338, 692 (1989)

7. Wilner T. Elementa 31, 13-24 (1948); 32, 28-31 (1949)

8. Söderberg, S. (ed.) Văr alkemist $i$ Tomegränd (Gleerup.
} Lund, 1970). 